\title{
Melanoma treatment with intratumoral electroporation of tavokinogene telseplasmid (plL-12, tavokinogene telseplasmid)
}

David A Canton ${ }^{1}$, Shawna Shirley ${ }^{1}$, Jocelyn Wright ${ }^{1}$, Richard Connolly ${ }^{1,2}$, Christoph

Burkart ${ }^{1}$, Anandaroop Mukhopadhyay ${ }^{1}$, Chris Twitty ${ }^{1}$, Kristen E Qattan ${ }^{1}$, Jean S Campbell², Mai H Le², Robert H Pierce², Sharron Gargosky¹, Adil Daud³ \& Alain Algazi*3

${ }^{1}$ OncoSec Medical Incorporated, 5820 Nancy Ridge Dr, San Diego, CA 92121, USA

${ }^{2}$ Fred Hutchinson Cancer Research Center, Clinical Research Division, 1100 Fairview Ave. N. Seattle, WA 98109, USA

${ }^{3}$ UCSF Helen Diller Family Comprehensive Cancer Center, 1600 Divisadero St, San Francisco, CA 94115, USA

* Author for correspondence: Alain.Algazi@ucsf.edu

Tumors evade detection and/or clearance by the immune system via multiple mechanisms. IL-12 is a potent immunomodulatory cytokine that plays a central role in immune priming. However, systemic delivery of IL-12 can result in life-threatening toxicity and therefore has shown limited efficacy at doses that can be safely administered. We developed an electroporation technique to produce highly localized IL-12 expression within tumors leading to regression of both treated and untreated lesions in animal models and in patients with a favorable safety profile. Furthermore, intratumoral tavokinogene telseplasmid electroporation can drive cellular immune responses, converting 'cold' tumors into 'hot' tumors. Clinical trials are ongoing to determine whether intratumoral tavokinogene telseplasmid electroporation synergizes with checkpoint blockade therapy in immunologically cold tumors predicted not to respond to PD-1 antibody monotherapy.

First draft submitted: 19 July 2017; Accepted for publication: 29 September 2017; Published online: 24 October 2017

Keywords: cancer immunotherapy • cytokine • electroporation • gene therapy • IL-12 • ImmunoPulse ${ }^{\circledR}$ IL-12 • ImmunoPulse ${ }^{\circledR}$ IT-tavo-EP • melanoma • pIL-12

\section{Immunotherapy in cancer}

Immunotherapy has recently gained momentum as a method for treatment of cancer in addition to surgery, chemotherapy and radiation therapy. Tumors are able to develop by evading host immunosurveillance, which occurs through a variety of mechanisms. Immuno-oncology research has focused on understanding mechanisms that promote antitumor immune responses while limiting tumor escape. One key discovery was that immune selfregulation through immune checkpoints, a process that limits autoimmunity, may be usurped during tumorigenesis. This led to the development of immune checkpoint inhibitors, which received US FDA approval in a variety of advanced solid tumors including metastatic melanoma, non-small-cell lung cancer, squamous carcinoma of the head neck and urothelial carcinoma [1-10]. Although immune checkpoint inhibitors have allowed large numbers of patients with otherwise incurable malignancies to achieve long-term remissions, often with limited side effects, response rates tend to be modest. For example, the objective response rate to PD-1 antibodies in metastatic melanoma is 33-40\% [6,7]. Correlative tissue analyses from clinical trials of PD-1 antibodies in metastatic melanoma patients have suggested that resistance or nonresponse to PD-1 antibodies may be seen in tumors with inadequate antitumor T helper type 1 (Th1) immune responses as shown by a reduced IFN- $\gamma$ signature [11], inadequate expression of inflammatory signals, poor infiltration with effector $T$ cells [12] or high levels of intratumoral regulatory cells [13].

Future Medicine 


\section{Exogenous administration of proinflammatory cytokines}

Exogenous administration of pro-inflammatory cytokines can induce intratumoral inflammation and recruitment of $\mathrm{T}$ cells, potentially leading to systemic immune activation. These agents would seem to be logical candidates to bridge the gap between ineffective and effective immune responses to PD-1 antibodies and they have been used for decades in advanced melanoma patients. IFN- $\alpha-2 \mathrm{a}$ is FDA-approved for the treatment of resected stage IIb through stage IIIc melanoma based on data suggesting that it improves relapse rates by approximately $12 \%$ [14-16]. However, there are no definitive data suggesting a survival benefit with this agent. High-dose IL-2 is FDA-approved for the treatment of metastatic melanoma and yields objective responses in approximately $17 \%$ of patients with durable remissions in approximately 3-5\% [17,18]. However, this agent has fallen out of favor because it must be administered in a monitored or ICU setting since it causes systemic inflammation leading to hypotension, capillary leak syndrome, and, potentially, multiorgan failure. Proinflammatory cytokines like IL-12 are elaborated locally in response to innate immune cell activation driven by 'danger signals' such as Toll-like receptor (TLR) agonists or other damage-associated molecular pattersn (DAMPs) and help to orchestrate a nuanced, coordinated immune reaction. As approved by the FDA, both IFN- $\alpha-2$ a and high-dose IL-2 are administered systemically, an approach that may lead to immunity confusion rather than immune cell recruitment and high levels of systemic toxicity.

\section{IL-12 as a cancer therapeutic}

IL-12 is a pleiotropic pro-inflammatory cytokine that activates the immune system via multiple mechanisms and is expressed by several immune cells including dendritic cells, macrophages and neutrophils [19]. IL-12 is a key Th1-polarizing cytokine along with IFN- $\gamma$ [20]. In addition to priming and maintaining Thelper and cytotoxic cell responses to specific antigens and the subsequent production and release of IFN- $\gamma$, IL-12 also has a role in activating natural killer cells, acts as an anti-angiogenic factor and it is important in the reactivation and survival of memory T cells [21]. Increasing intratumoral IL-12 may increase the immune response against cancer cells. Furthermore, IL-12 has also been shown to improve the immune response by inhibiting regulatory $\mathrm{T}$ cells [22], Th2 responses [23] and myeloid-derived suppressor cells [22,24,25].

The IL-12 cytokine has been the subject of many clinical studies to determine its safety and efficacy as an antitumor agent, and, like IFN- $\alpha-2 \mathrm{a}$ and high-dose IL-2, systemic administration of IL-12 has been associated with limited efficacy and substantial toxicity [21,26-33]. Direct administration of IL-12 into solid tumors induces a switch from a Th2 to a Th1 intratumoral immune profile in a surgical window study, but this approach is again associated with significant treatment-associated toxicity [34]. In a separate Phase 1/1B clinical trial, intralesional IL-12 leads to regression of injected lesions by at least 30\% in five of 12 patients treated. However, there was no evidence of clinically meaningful systemic immune activation since regression of nontreated lesions was not observed in any patient [35]. Similar findings have been observed in patients treated with intralesional IL-2, which leads to frequent regressions of treatment lesions but not abscopal regressions of untreated lesions [36,37]. These findings are interesting but inadequate since the goal of cytokine therapy is to induce systemic immune changes that alone or in combination with additional agents could lead to durable remissions in cancer patients.

\section{Electroporation of IL-12 DNA to enhance therapeutic delivery in preclinical models}

Electroporation has been shown to be a safe and effective method to deliver small molecules directly to target tissues. The technique was developed in the early 1980s as a method of transfecting cells in vitro [38]. Technological advances and decades of research have led to its use in delivering chemotherapeutic agents to patients for tumor ablation, a procedure called electrochemotherapy [39]. Reversible electroporation, which uses lower energy levels to transiently permeabilize the cell membrane, is also being investigated in clinical studies for the delivery of nucleic acids to tissues to modulate patient's immune responses [40].

There is a large body of preclinical data in support of the use of electroporation of IL-12 DNA followed by electroporation as an anticancer therapeutic. Electroporation of pIL-12 DNA has been demonstrated to yield high levels of IL-12 expression [41] leading to IFN- $\gamma$-mediated tumor cell killing [42,43], immune cell recruitment [41] and objective tumor regressions in animal models [43-45] including regression of untreated lesions [46]. In vivo tumor regressions have been durable and treated animals may be resistant to tumor reimplantation suggesting that intratumoral electroporation of pIL-12 DNA induces memory immune responses in these models [47,48]. Although intramuscular electroporation of pIL-12 has been examined, intratumoral therapy leads to more elaboration of cytokines in the tumor microenvironment and higher rates of tumor regressions [49,50]. 


\section{Electroporation of IL-12 DNA in human subjects}

Although electroporation of IL-12 DNA has been tested extensively in preclinical models, data supporting this approach in human subjects, particularly in the context of malignancy, were limited until recently. Intramuscular electroporation of IL-12 plasmid in conjunction with HIV vaccination was found to be well tolerated in human subjects and potentially improved antigenicity and immunicity [51-53].

\section{INO-9012}

The IL-12 delivery system with electroporation INO-9012 has been shown to be well tolerated as an adjuvant to tumor vaccination in prostate cancer [54], although efficacy data are limited. INO-9012 is also currently in testing in combination with INO-1400, an agent targeting human telomerase reverse transcriptase, in patients with advanced solid tumors [55].

\section{Intratumoral tavokinogene telseplasmid electroporation (ImmunoPulse ${ }^{\circledR}$ IL-12)}

Tavokinogene telseplasmid (tavo) (IT-tavo-EP) has recently been granted orphan drug designation for unresectable metastatic melanoma by the FDA. Intratumoral injection of plasmid IL-12 pUMVC3-hIL-12-NGVL3 followed by electroporation (IT-tavo-EP) results in highly localized IL-12 expression and very-low systemic doses of IL12. Clinical data to date demonstrate that IT-tavo-EP acts as an in situ tumor vaccine by inducing local IL-12 expression which, in turn, leads to intratumoral inflammation and tumor antigen presentation, recruitment of $\mathrm{T}$ cells, enhanced Th1 immune responses, and, importantly, systemic immune response with minimal systemic toxicity $[13,56,57]$. These systemic, biological effects of IT-tavo-EP could be synergistic with the activities of PD-1 and PD-L1 antibodies, and clinical trials are ongoing to formally evaluate this potential synergy.

The plasmid IL-12 used in the initial Phase I trial [58] has been termed tavo. Tavo (Figure 1A) is a plasmid that encodes genes for both the $\mathrm{p} 35$ and $\mathrm{p} 40$ subunits of the heterodimeric human IL-12 protein separated by an internal ribosome entry site. Constitutive expression of the subunits is driven by a single cytomegalovirus promotor. Once the plasmid is introduced into a mammalian cell, a functional IL-12p70 protein is expressed and secreted into the tumor microenvironment. Intratumoral electroporation of accessible lesions with tavo (IT-tavo-EP) is performed using an in situ electroporation device.

The current device in clinical use consists of two main components used for electroporation (Figure 1B). An electrode applicator with six sterile disposable trocar electrodes is arranged in a circular array, which is controlled by the electric pulse generator. The electroporation generator incorporates solid-state switched capacitive output and a built-in auto-switching capability. This switching feature automatically sequences the various needle electrodes so that the appropriate electric field is applied during the therapy process. The therapy pulse output shape is in the form of a square wave. The pulse length is of a fixed duration; pulse amplitude and needle array sequencing mode are automatically controlled by the generator after docking with the handle, which contains a chip encoding the EP parameters. Additional device specifications are shown in Table 1.

\section{Administration}

Tavo is directly injected into a tumor lesion using a standard hypodermic needle. The six electrodes of the EP applicator are then inserted into the lesion and the electroporation pulses applied to locally permeabilize and transduce the cells.

The generator delivers a total of six $1500 \mathrm{~V} / \mathrm{cm}$ pulses where each pulse is $100 \mu$ s in duration with $300 \mathrm{~ms}$ intervals between pulses. Pulses are applied in pairs; each pulse passes from a pair of electrodes to the opposing pair. After the initial pulse in the sequence, the electrode polarity is reversed to deliver the second pulse in the pair. Pulse delivery is then rotated clockwise to the next opposing pair of needle electrodes and pulse pairs are applied until all six pulses in the sequence are applied. In the current study protocols, patients receive a series of treatment cycles of IT-tavo-EP at 6-week intervals. Each cycle consists of three administrations, one on each Day 1,5 and 8 , at a dose volume of approximately one quarter the calculated lesion volume at a plasmid concentration of $0.5 \mathrm{mg} / \mathrm{ml}[45,57,58]$.

IT-tavo-EP is designed to produce local expression of IL-12 protein within the tumor. The application of electroporation pulses allows tavo to enter the cells, then these cells express and secrete IL-12 protein, which initiates a local immune response (Figure 1C). Only cells that are simultaneously exposed to both tavo plasmid DNA and the generated electrical field are transfected. This ensures local, targeted delivery of the plasmid and facilitates local secretion of IL-12. 
(A)

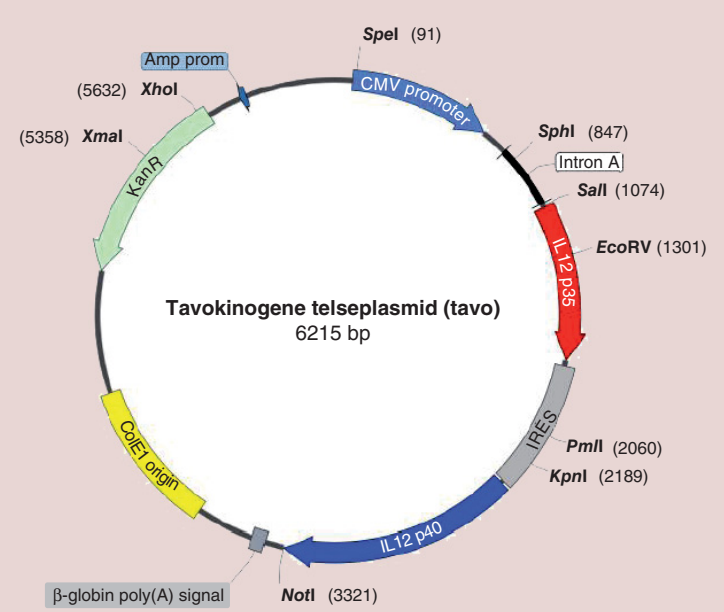

(B)

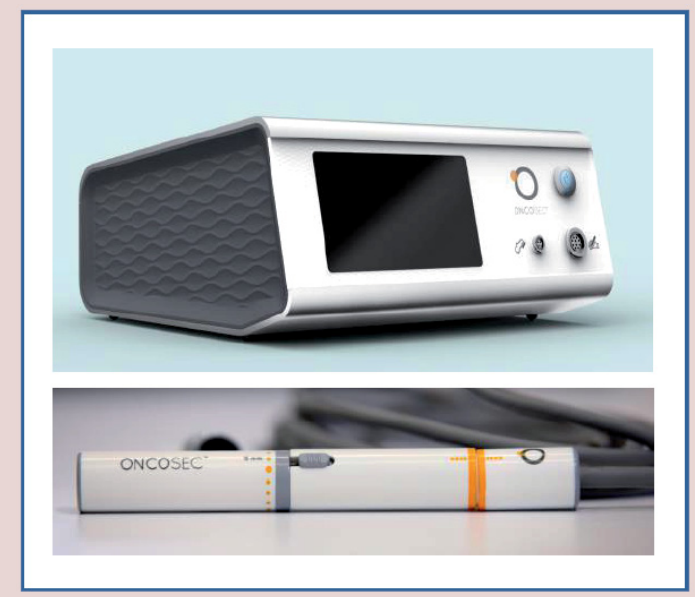

(C)

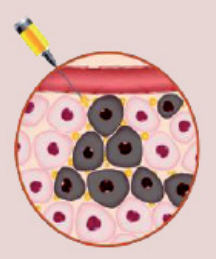

(1)

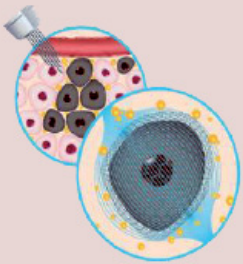

(2)

\begin{abstract}
Intratumoral
\end{abstract}
EP delivers tavo into cells

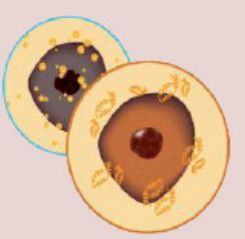

(3)

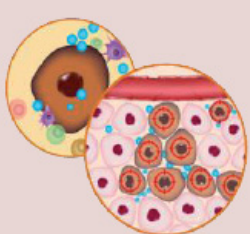

(4)

Local inflammation and $T$ cell education

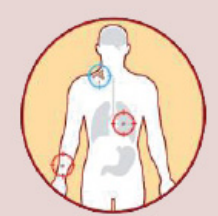

(5)

Systemic anti-tumor immune response

Figure 1. Overview of intratumoral tavokinogene telseplasmid electroporation therapy.

Tavo-EP therapy delivers (A) tavokinogene telseplasmid (tavo; plasmid IL-12; pIL12) directly to tumors using the (B) OncoSec medical system (OMS ${ }^{\circledR}$ ) electroporation platform, which consists of an electroporation generator (top) and a depth adjustable electrode applicator with disposable trocar electrodes (bottom). (C) Tavo is directly injected into a tumor lesion and electroporation pulses are applied to locally permeabilize and transduce the cells. Upon transfection, cells express and secrete IL-12p70 protein into the tumor microenvironment initiating local and systemic immune responses.

EP: Electroporation; IT: Intratumoral.

\section{Preclinical studies}

The antitumor effects of IL-12 have previously been evaluated in a variety of preclinical models using the murine homolog of the cytokine [59]. It has been shown that the antitumor efficacy of IL-12 gene therapy is comparable to IL-12 recombinant protein therapy with far fewer undesirable side effects [60]. Preclinical studies to evaluate the effects of electroporation of plasmid IL-12 (pIL-12-EP) were carried out in a syngeneic mouse model using poorly immunogenic and highly metastatic B16.F10 melanoma tumor model. Administration of intratumoral pIL-12$\mathrm{EP}$ as a single treatment cycle was shown to significantly delay tumor growth [61]. Complete tumor regression was achieved with two and three treatment cycles [47,62]. Mice treated with pIL-12-EP experienced prolonged survival, and in some studies showed complete regression and remained disease-free for 50 days post treatment. No significant increase in serum levels of IL-12 was identified. Rechallenge with B16.F10 cells in these studies indicate the generation of a systemic antitumor memory response. 
Table 1. OMS ${ }^{\circledR}$ electroporation generator specifications (operator's manual).

Treatment specifications

Electroporation duration

Pulses per electroporation sequence

Technical specifications

Output pulse voltage range

Output pulse current protection

Output pulse pattern

Nominal input voltage requirements

Input power voltage range

Input power current

Overcurrent protection

Physical specifications

Weight

Dimensions

Connectors

\section{$<10 \mathrm{~s}$}

Six pulses

400-1500 VDC

$400 \mathrm{~A}$ max, $10 \mu \mathrm{s}$ max duration

Square wave, $100 \mu$ s duration

Selectable from $100,120,220$ or 240 VAC RMS $50 / 60 \mathrm{~Hz}$

-20 to $+10 \%$ of the nominal voltage

2.3 A RMS (at 120 VAC RMS) typical

1.6 A at $250 \mathrm{~V}$ (Type T, IEC 127, slow blow, $5 \times 20 \mathrm{~mm}$ )

$10 \mathrm{~kg}$

$16 \mathrm{~cm}$ (height) $\times 40 \mathrm{~cm}$ (width) $\times 42 \mathrm{~cm}$ (depth)

12 pin, indexed, locking connection to OMS applicator

4 pin, indexed, locking connection to foot switch

9 pin, standard connection to OMS printer

3 pin with ground, standard power input module for AC power cord

AC: Alternating current; IEC: International electrotechnical commission; OMS: OncoSec medical system; RMS: Root mean square; VAC: Volts alternating current; VDC: Volts direct current.

These antitumor effects of pIL-12-EP have been shown to be associated with a local influx of activated cytotoxic T lymphocytes and partially dependent on IFN- $\gamma$, granzyme and perforin [42]. This study also demonstrated generation of $\mathrm{CD}^{+} \mathrm{T}$ cells specific to tumor-associated antigen tyrosinase-related peptide $2(\operatorname{Trp} 2)$.

To test for the systemic effects of IT-tavo-EP, we created a version of tavo containing the murine IL-12 homolog and utilized a syngeneic, contralateral tumor model. Briefly, C57BL/6 mice were injected with $1 \times 10^{6} \mathrm{~B} 16 . \mathrm{F} 10$ tumor cells on both the primary (treated) and contralateral (untreated) side. Once the primary tumors reached a treatable volume, tavo DNA was injected and electroporated with one treatment cycle consisting of three applications of IT-tavo-EP (Days 1,4 and 7). Tumor volume $\left(\mathrm{V}=\mathrm{a}^{2} \mathrm{~b} / 2\right.$, where $\mathrm{a}$ is the shortest diameter and $\mathrm{b}$ is the longest diameter) was measured on both the treated primary lesion and the untreated contralateral tumor, allowing the discernment of local and systemic effects. As shown in Figure 2, IT-tavo-EP caused significant delay in growth of both the treated (Figure 2A) and untreated (Figure 2B) tumors. By immunohistochemistry, there was an increase in immune cell infiltrate in treated and untreated lesions post treatment (Figure 3A \& B). A total of 48-h post EP, tumors were excised, homogenized and assayed for IL-12p70 and IFN- $\gamma$ by ELISA (mouse IL-12p70 and IFN- $\gamma$ DuoSet ELISAs, R\&D Systems, MN, USA). IT-tavo-EP increased IL-12 and IFN- $\gamma$ levels in both treated and untreated tumors (Figure 3C, left and middle panels). Serum levels of IL-12 were not significantly different than untreated controls confirming local IL-12 expression (Figure 3C, right panel). IFN- $\gamma$ levels however were slightly elevated in the serum, which may reflect the triggering of a systemic immune activation.

Collectively, our studies and multiple literature reports indicate that intratumoral pIL-12-EP can generate local and systemic immune responses that can convert immunologically cold tumors to T-cell inflamed hot tumors. These preclinical data supported the initiation of a clinical program to assess the safety and efficacy of IT-tavo-EP as a monotherapy in patients with advanced melanoma with accessible lesions for electroporation therapy (Table 2).

In order to move the approach to clinical testing, a preclinical toxicity study was performed [45]. Tumors were established in the left flank of $\mathrm{C} 57 \mathrm{Bl} / 6$ mice and divided into control and treated groups. Three treatments were performed on days 1, 5 and 8. Extensive hematology and serum chemistry analysis was performed and mice were sacrificed on days 9, 11, 16, 23 and 30 after treatment. There was decreased survival of untreated mice compared with partially treated (plasmid only, no electrotransfer) or treated mice (plasmid plus electrotransfer). All deaths were due to disease progression. No weight loss was seen in any of the groups. Histological examination of the heart, lungs, brain, bone, muscle and liver showed no evidence of abnormalities correlated with treatment, although there was inflammation noted in kidneys examined from some animals treated with both IL-12 plasmid and electroporation. Mice in the group receiving plasmid IL-12 delivered by electroporation were generally in the best health and showed the least biochemical and hematology abnormalities. 


\section{(A)} IT-tavo-EP treated tumor
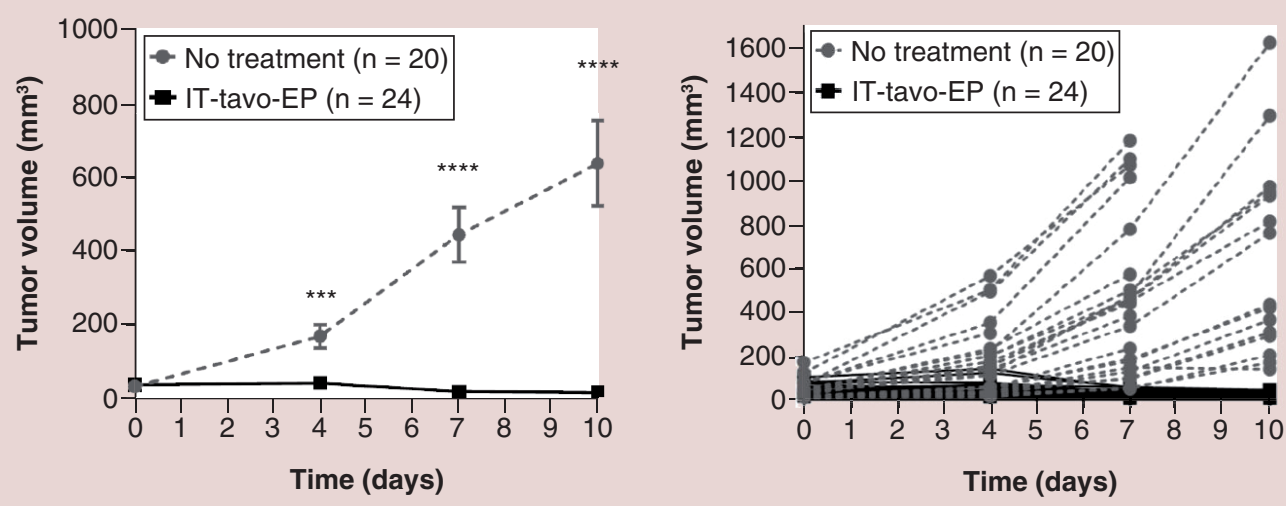

(B)

Untreated tumor
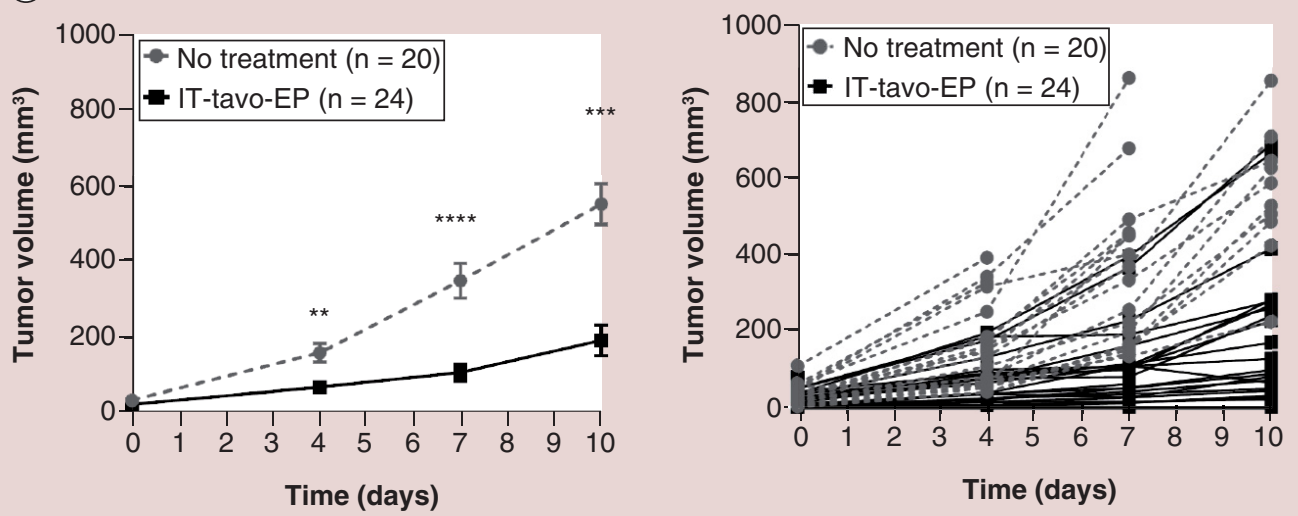

Figure 2. Tumor growth delay in a preclinical B16.F10 melanoma model.

Intratumoral tavokinogene telseplasmid electroporation resulted in significant delay in growth of (A) treated and (B) untreated tumors in a contralateral B16.F10 murine melanoma model. C57BL/6 mice were injected at two sites with $1 \times 106$ B16.F10 tumor cells on both the primary (treated) and contralateral (untreated) side. Once the mean primary tumor size reached a treatable size range, it was injected with tavo DNA and electroporated with one treatment cycle consisting of three applications of IT-tavo-EP (Days 1, 4 and 7). Both treated and untreated tumors were measured using digital vernier calipers and the volume calculated using the formula $V=a 2 b / 2$, where $a$ is the shortest diameter and $b$ is the longest diameter. The panels on the left show consolidated tumor growth curves; the panels on the right show growth curves for each individual mouse as spaghetti plots. Significance was calculated using an unpaired two-tailed Mann-Whitney test. ${ }^{* *} \mathrm{p} \leq 0.01,{ }^{* * *} \mathrm{p} \leq 0.001$ and $* * * * \mathrm{p} \leq 0.0001$.

EP: Electroporation; IT: Intratumoral; Tavo: Tavokinogene telseplasmid.

\section{A first-in-human Phase I trial of tavo electroporation}

A phase I trial was conducted at the Moffitt Cancer Center from December 2004 to February 2007 [49]. Patients had metastatic melanoma with accessible cutaneous disease that was surgically unresectable. Twenty-four patients were treated in seven cohorts (plasmid concentrations ranging from 0.1 to $1.6 \mathrm{mg} / \mathrm{ml}$ ). All patients had three treatments on days 1, 5 and 8 which included intratumoral injection of tavo plasmid delivered using electroporation at six pulses $100 \mu$ s long and $1500 \mathrm{~V} / \mathrm{cm}$. No systemic treatment-related adverse events (including nephrotoxicity) were observed. Transient pain, erythema and bleeding at the electroporation sites following treatment were the only adverse effects reported but all patients stated that the therapy was tolerable. Although the study was designed as a safety trial, over $70 \%$ of the treated lesions regressed. A total of 19 of the 24 patients enrolled in this study had additional sites of disease outside the treated lesions. Of these 19 patients, $10 \%$ showed complete regression of all metastases, both treated and untreated, and $42 \%$ showed disease stabilization or partial response. Post-treatment biopsies at various times after treatment (Days 11, 22 and 39) showed significant increases in IL-12 protein associated 
(A)

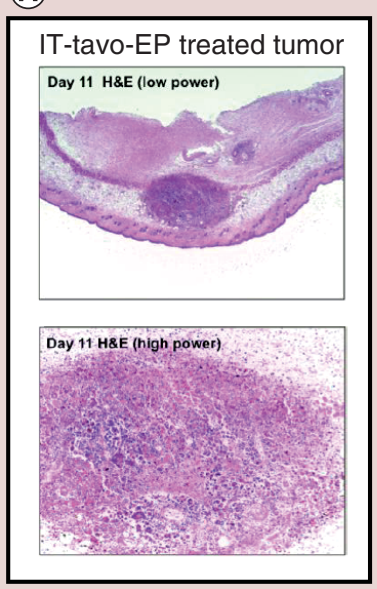

(B)

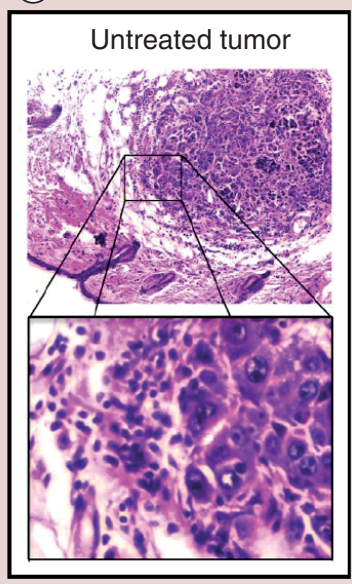

(C) Treated tumor
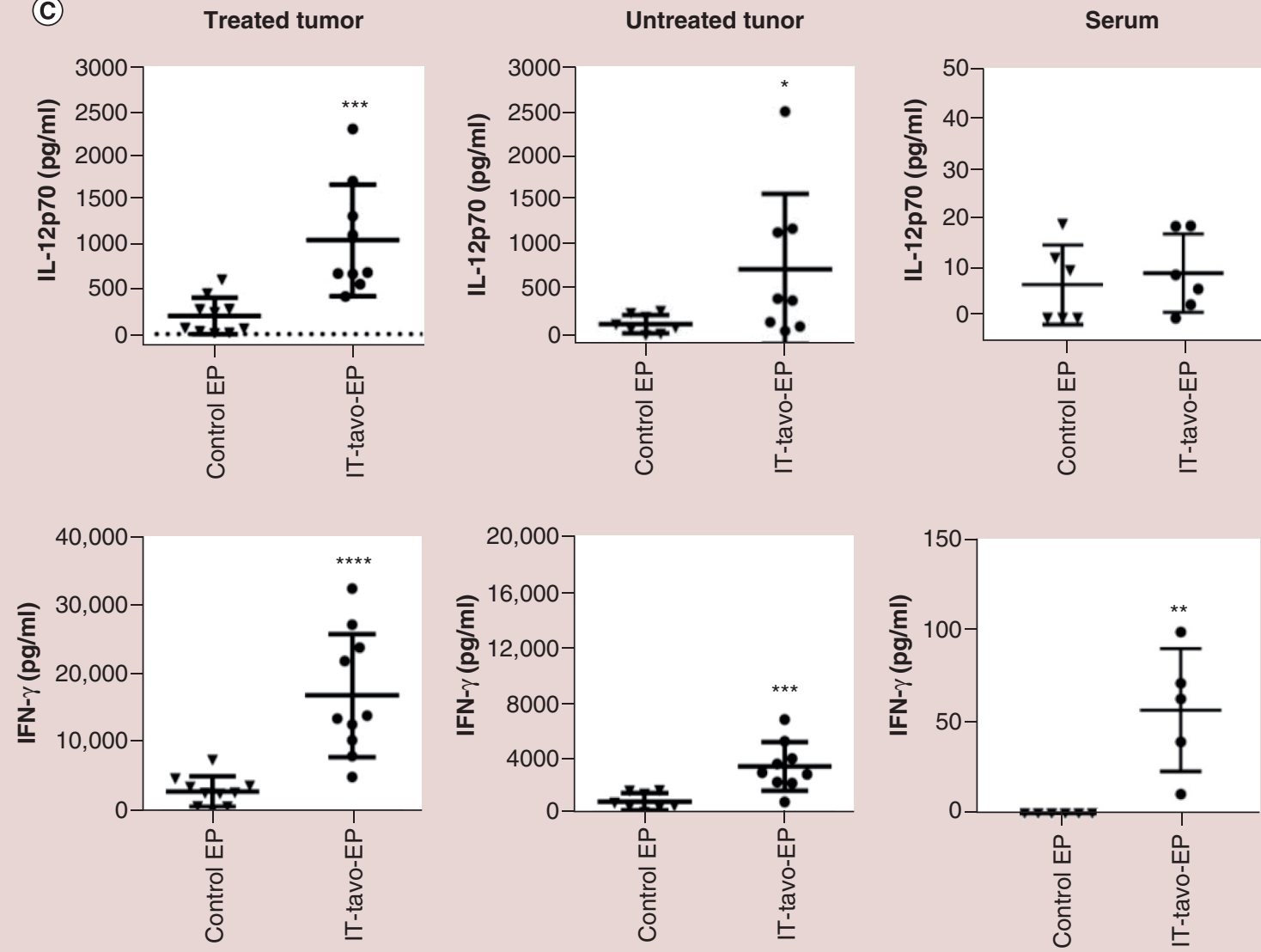

Figure 3. Intratumoral tavokinogene telseplasmid electroporation increased immunogenicity in B16.F10 tumors. Treated tumors $(5 / 5)$ regressed and demonstrated $(A)$ extensive necrosis and complete pathological response. A total of 18 days after treatment, untreated tumors exhibited (B) immune cell infiltration. IT-tavo-EP induced (cleft) IL-12 and (C - middle) IFN- $\gamma$ protein expression in both treated and untreated tumors as shown by ELISA. No detectable (C right) IL-12 was observed in the serum, but IFN- $\gamma$ was found to be elevated in animals treated with IT-tavo-EP. Significance was calculated using an unpaired two-tailed Mann-Whitney test.

${ }^{*} \mathrm{p} \leq 0.05, * * \mathrm{p} \leq 0.01, * * * \mathrm{p} \leq 0.001$ and $* * * * \mathrm{p} \leq 0.0001$

EP: Electroporation; H\&E: Hematoxylin and eosin stain; IT: Intratumoral; Tavo: Tavokinogene telseplasmid. 
Table 2. Intratumoral tavokinogene telseplasmid electroporation clinical studies in patients with advanced

\section{melanoma.}

\begin{tabular}{|c|c|c|c|c|}
\hline & Title & Study purpose & Enrollment & Ref. \\
\hline Phase I & $\begin{array}{l}\text { Phase I trial of intratumoral plL-12 electroporation in } \\
\text { malignant melanoma }\end{array}$ & $\begin{array}{l}\text { Single-dose escalation study to determine the safety and } \\
\text { tolerability of IT-tavo-EP }\end{array}$ & 24 & [58] \\
\hline Phase II & $\begin{array}{l}\mathrm{OMS}^{\circledR}-1100 \text { : trial of plL-12 electroporation malignant } \\
\text { melanoma }\end{array}$ & $\begin{array}{l}\text { To assess safety, tolerability and explore the antitumor } \\
\text { activity of IT-tavo-EP }\end{array}$ & 51 & [65] \\
\hline Phase II & $\begin{array}{l}\text { OMS-1102: a multicenter Phase II study of enhancing } \\
\text { pembrolizumab responses in melanoma through } \\
\text { intratumoral pIL-12 electroporation }\end{array}$ & $\begin{array}{l}\text { To assess the antitumor efficacy (defined as the best } \\
\text { overall response rate using RECIST v1.1) of the } \\
\text { combination of IT-tavo-EP and pembrolizumab in patients } \\
\text { with low TIL melanoma (Figure 5) }\end{array}$ & 22 & [66] \\
\hline Phase II & $\begin{array}{l}\text { OMS-I103: a multicenter Phase II, adaptive, open-label trial } \\
\text { of intratumoral pIL-12 plus electroporation in combination } \\
\text { with pembrolizumab in patients with stage III/IV } \\
\text { melanoma who are progressing on either pembrolizumab } \\
\text { or nivolumab }\end{array}$ & $\begin{array}{l}\text { To assess the efficacy, safety and tolerability up to } \\
24 \text { weeks of combination therapy of IT-tavo-EP and } \\
\text { pembrolizumab (iv.) in patients with stage III/IV } \\
\text { melanoma with disease progression on or within } 24 \text { weeks } \\
\text { of receiving prior anti-PD-1 agents (e.g., pembrolizumab } \\
\text { and nivolumab) }\end{array}$ & 48 (planned) & [66] \\
\hline
\end{tabular}

EP: Electroporation; IT: Intratumoral; OMS ${ }^{\circledR}$ : OncoSec medical system; Tavo: Tavokinogene telseplasmid; TIL: Tumor-infiltrating lymphocytes.

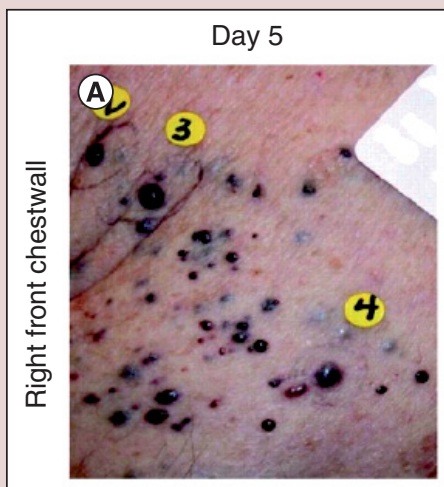

\section{Day 256}

(B).
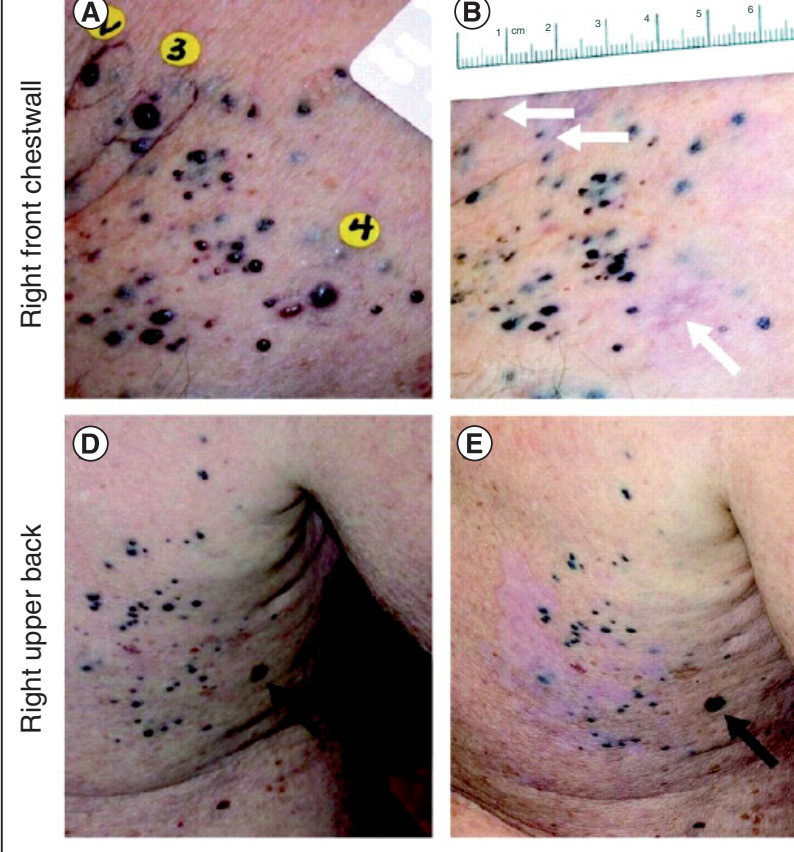

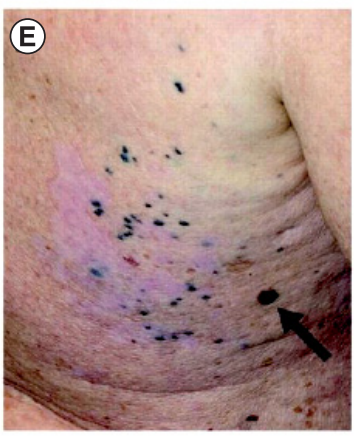

Day 637
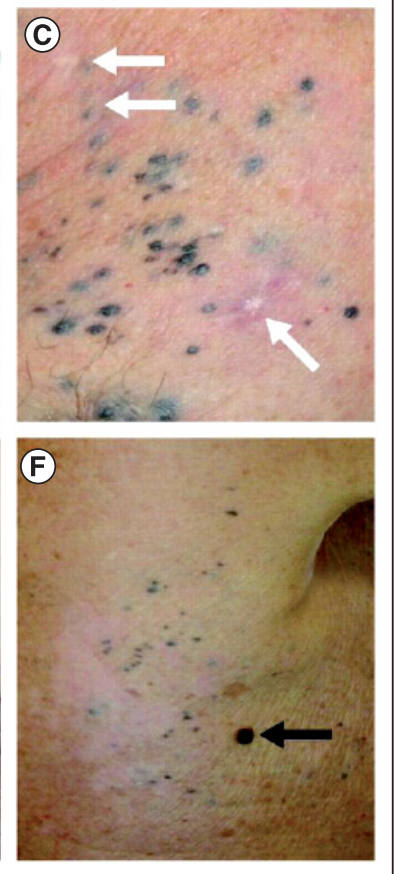

Figure 4. Response over 18 months in patient treated with intratumoral tavokinogene telseplasmid electroporation. Only the numbered lesions were treated. Biopsied lesions at 7 and 18 months showed melanin pigment without evidence of melanoma. Black arrows indicate a seborrheic keratosis for reference.

Reproduced with permission from [58] ( ) American Society of Clinical Oncology (2008). All rights reserved.

with plasmid electroporation. Marked tumor necrosis and lymphocytic infiltrate were detected histologically. The results from this study show that IT-tavo-EP delivery to melanoma patients is safe and capable of inducing an effective antitumor immune response. Two patients showed long-term durable complete response in both treated and untreated lesions. In one patient who had rapidly progressing cutaneous metastasis with over 50 nodules on his right chest and shoulder, following electroporation, no new lesions developed and over a period of 18 months all lesions flattened out and faded (Figure 4) [58]. A sample regressed lesion was biopsied and demonstrated residual pigment but no evidence of melanoma. He has no evidence of systemic disease 6 years following treatment. A third 


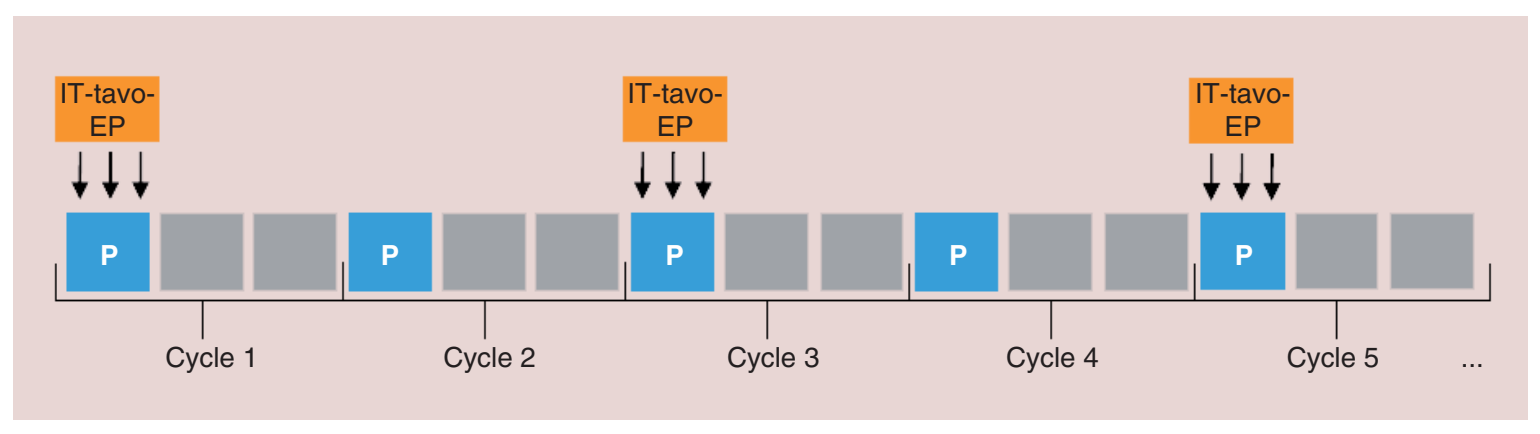

Figure 5. OncoSec medical system (OMS)-I102 Phase II trial design: intratumoral tavokinogene telseplasmid electroporation in combination with pembrolizumab in predicted pembrolizumab nonresponders.

Patients were enrolled based on $<25 \%$ CTLA4hiPD1hi TIL phenotype. Pembrolizumab (P) was administered at a fixed dose of $200 \mathrm{mg}$ iv. over 30 min every 3 weeks. Patients were treated with IT-tavo-EP on days 1, 5 and 8 of every other cycle (every 6 weeks).

EP: Electroporation; IT: Intratumoral; Tavo: Tavokinogene telseplasmid.

patient, had complete regression of all lesions (treated and untreated) and on a follow-up CT scan had no evidence of disease 5 months post electroporation, after having received four cycles of dacarbazine.

\section{Phase II clinical trial data}

To further explore the effectiveness of tavo electroporation, a single arm, multicenter, open label Phase II trial (OMS-I100) was carried out in patients with stage III and IV melanoma with accessible metastasis. The primary end point was the tumor response as defined by modified skin Response Evaluation Criteria in Solid Tumors (msRECIST), which permits inclusion of visible cutaneous metastases measuring greater than $3 \times 3 \mathrm{~mm}$ as target lesions. This trial enrolled 51 patients who received at least one treatment cycle, with three administrations of ITtavo-EP $(0.5 \mathrm{ml}$ of $0.5 \mathrm{mg} / \mathrm{ml}$ for a fixed dose of $0.25 \mathrm{mg}$ for each injectable tumor treatment zone) on either days 1,5 and 8 or on days 1,8 and 15 of each treatment cycle. Between two and four lesions accessible to electroporation were treated per patient per cycle (each lesion was treated with three treatments per cycle). Disease evaluation was carried out at 90-day intervals. If there was evidence of persistent disease and the disease was determined to not have progressed, patients received up to a total of four additional treatment cycles over the course of 360 days. Investigators were requested to maintain one untreated lesion for the duration of the trial to enable the assessment of systemic antitumor immune responses.

PD-L1 expression and expression of an IFN- $\gamma$ gene signature, which has been associated with increased PD-L1 expression, have been identified as positive predictor of response to PD-1 and PD-L1 antibody therapy [11,63,64]. IT-Tavo-EP has been shown to increase IFN- $\gamma$ gene expression potentially priming less inflamed tumors to respond to PD-1 and PD-L1 antibodies. Two additional studies have been initiated to address the safety and efficacy of the combination of IT-tavo-EP and pembrolizumab: a multicenter Phase II study (OMS-I102) in stage III-IV melanoma patients with low TIL status (Figure 5), and a multicenter Phase II study (OMS-I103) in patients who are progressing on either pembrolizumab or nivolumab. In addition, patients enrolled in OMS-I100 were tracked after completion of IT-tavo-EP (OMS-I100 retrospective). Formal manuscripts from these studies are in preparation and publication of these results is anticipated within the next few months.

\section{Conclusion}

Collectively, preclinical and Phase I clinical trial data demonstrate that IT-tavo-EP is safe and well tolerated by patients, unlike systemically administered cytokine therapy with IL-12 or high-dose IL-2. Unlike intratumoral cytokine protein (as opposed to plasmid DNA) administration, IT-tavo-EP induces regression of untreated lesions suggesting that this approach acts as an in situ tumor vaccination inducing systemic antitumor immunity.

Inhibition of the PD-1/PD-L1 axis with monoclonal antibodies has changed the therapeutic landscape for melanoma patients. Unfortunately, many patients fail to respond to these new therapies. This patient population of PD-1 nonresponders represents an unmet medical need that many researchers are currently trying to address with novel combination therapies. Because IT-tavo-EP increases intratumoral lymphocyte infiltration, pro-inflammatory cytokines and Th1 immune responses, it could potentiate the activity of PD-1 antibodies without adding significant 
systemic toxicity. Formal clinical trial data examining the potential synergy between IT-tavo-EP and PD-1 antibodies in advanced melanoma patients will be anticipated within the next few months.

Executive summary

Background

- IL-12 is a potent immune activator studied extensively as an anticancer agent.

- Toxicity of systemic administration of IL-12 precludes significant anticancer efficacy.

- US FDA-approved therapeutic antibodies inhibit PD-1/PD-L1 and CTLA-4 immune checkpoint pathways show durable responses in patients with metastatic melanoma.

- Despite this success, the majority of patients fail to respond to checkpoint blockade representing an unmet medical need.

Overview of intratumoral tavokinogene telseplasmid electroporation (Immunopulse ${ }^{\circledR}$ IL-12)

- Tavokinogene telseplasmid (tavo) is a DNA plasmid that encodes genes for the p35 and p40 subunits of human IL-12.

- Tavo is injected into the lesion and electroporation pulses applied using an electrode applicator with disposable needle electrodes and an electric pulse generator.

- Transfected cells express and secrete IL-12 protein, which initiates a local immune response.

Preclinical studies

- Intratumoral electroporation of tavo into B16.F10 tumors increased IL-12p70 in the tumor microenvironment but not in serum.

- Studies performed in a two tumor B16.F10 murine melanoma model show tavo-induced control of growth of both treated and untreated lesions.

Clinical studies

- Intratumoral tavokinogene telseplasmid electroporation (IT-tavo-EP) monotherapy has been tested in Phase I and Phase II trials in metastatic melanoma and has proven to be safe and well tolerated.

- IT-tavo-EP therapy increases lymphocyte infiltration and local IFN- $\gamma$ expression in patients' tumors.

- An Phase II trial testing IT-tavo-EP in combination with systemic pembrolizumab therapy in patients predicted to be pembrolizumab nonresponders is ongoing.

- Tavo has recently been granted orphan drug designation by the FDA for treatment of unresectable metastatic melanoma.

Conclusion

- Intratumoral expression of IL-12 immunologically reshapes tumor microenvironment, driving immunologically 'cold' tumors to immunologically 'hot'.

- IT-tavo-EP generates local and systemic antitumor immune effects leading to inhibition or clearance of treated and nontreated lesions.

- Collectively, current data indicate that IT-tavo-EP can effectively prime a cellular immune response that augments the efficacy of anti-PD-1 therapy.

- Phase II trial data of IT-tavo-EP alone and in combination with pembrolizumab will be formally reported soon.

Financial \& competing interests disclosure

S Shirley, J Wright, R Connolly, C Burkart, A Mukhopadhyay, KE Qattan, S Gargosky and DA Canton are current employees of Oncosec Medical Incorporated. DA Canton, R Connolly, JS Campbell and RH Pierce are inventors on patents, pending and published. RH Pierce is the former CSO of OncoSec and has a financial interest. MH Le is the former CMO of OncoSec and has a financial interest. A Daud acts as a Senior Advisor to OncoSec and has a financial interest. A Algazi has no personal financial interest in OncoSec, but he serves as Principal Investigator for clinical trial funded by OncoSec and he serves as an unpaid member of OncoSec's Scientific Advisory Board. The authors have no other relevant affiliations or financial involvement with any organization or entity with a financial interest in or financial conflict with the subject matter or materials discussed in the manuscript apart from those disclosed.

No writing assistance was utilized in the production of this manuscript.

\section{References}

Papers of special note have been highlighted as: $\bullet$ of interest; $\bullet \bullet$ of considerable interest

1 Brahmer J, Reckamp KL, Baas P et al. Nivolumab versus docetaxel in advanced squamous-cell non-small-cell lung cancer. N. Engl. J. Med. 373(2), 123-135 (2015). 
2 Fehrenbacher L, Spira A, Ballinger M et al. Atezolizumab versus docetaxel for patients with previously treated non-small-cell lung cancer (POPLAR), a multicentre, open-label, Phase II randomised controlled trial. Lancet 387(10030), 1837-1846 (2016).

3 Garon EB, Rizvi NA, Hui R et al. Pembrolizumab for the treatment of non-small-cell lung cancer. N. Engl. J. Med. 372(21), 2018-2028 (2015).

4 Seiwert TY, Burtness B, Mehra R et al. Safety and clinical activity of pembrolizumab for treatment of recurrent or metastatic squamous cell carcinoma of the head and neck (KEYNOTE-012), an open-label, multicentre, Phase Ib trial. Lancet Oncol. 17(7), 956-965 (2016).

5 Ferris RL, Blumenschein G, Fayette J et al. Nivolumab for recurrent squamous-cell carcinoma of the head and neck. N. Engl. J. Med. 375, 1856-1867 (2016).

6 Robert C, Long GV, Brady B et al. Nivolumab in previously untreated melanoma without BRAF mutation. N. Engl. J. Med. 372(4), $320-330$ (2015).

7 Robert C, Schachter J, Long GV et al. Pembrolizumab versus ipilimumab in advanced melanoma. N. Engl. J. Med. 372(26), 2521-2532 (2015).

8 Bellmunt J, de Wit R, Vaughn DJ et al. Pembrolizumab as second-line therapy for advanced urothelial carcinoma. N. Engl. J. Med. 376(11), 1015-1026 (2017).

9 Rosenberg JE, Hoffman-Censits J Atezolizumab in patients with locally advanced and metastatic urothelial carcinoma who have progressed following treatment with platinum-based chemotherapy: a single-arm, multicentre, Phase II trial. Lancet 387(10031), 1909-1920 (2016).

10 Sharma P, Retz M, Siefker-Radtke A et al. Nivolumab in metastatic urothelial carcinoma after platinum therapy (CheckMate 275), a multicentre, single-arm, Phase II trial. Lancet Oncol. 18(3), 312-322 (2017).

11 Ayers M, Lunceford J, Nebozhyn M et al. IFN- $\gamma$-related mRNA profile predicts clinical response to PD-1 blockade. J. Clin. Invest. 127(8), 2930-2940 (2017).

12 Tumeh PC, Harview CL, Yearley JH et al. PD-1 blockade induces responses by inhibiting adaptive immune resistance. Nature 515(7528), 568-571 (2014).

- Demonstrated that baseline signs of adaptive immune resistance are associated with response to PD-1 antibodies suggesting that pro-inflammatory signals could potentiate responses to PD-1 antibodies.

13 Algazi AP, Tsai KK, Rosenblum M et al. Immune monitoring outcomes of patients with stage III/IV melanoma treated with a combination of pembrolizumab and intratumoral plasmid interleukin 12 (pIL-12). J. Clin. Oncol. 35(Suppl. 7S), Abstract 78 (2017).

14 Eggermont AMM, Suciu S, Santinami M et al. Adjuvant therapy with pegylated interferon alfa-2b versus observation alone in resected stage III melanoma: final results of EORTC 18991, a randomised Phase III trial. Lancet 372(9633), 117-126 (2008).

15 Kirkwood JM, Strawderman MH, Ernstoff MS, Smith TJ, Borden EC, Blum RH. Interferon alfa-2b adjuvant therapy of high-risk resected cutaneous melanoma: the Eastern Cooperative Oncology Group Trial EST 1684. J. Clin. Oncol. 14(1), 7-17 (1996).

16 Kirkwood JM, Manola J, Ibrahim J et al. A pooled analysis of eastern cooperative oncology group and intergroup trials of adjuvant high-dose interferon for melanoma. Clin. Cancer Res. 10(5), 1670-1677 (2004).

17 Rosenberg SA, Yang JC, Topalian SL et al. Treatment of 283 consecutive patients with metastatic melanoma or renal cell cancer using high-dose bolus interleukin 2. JAMA 271(12), 907-913 (1994).

18 Atkins MB, Lotze MT, Dutcher JP et al. High-dose recombinant interleukin 2 therapy for patients with metastatic melanoma: analysis of 270 patients treated between 1985 and 1993. J. Clin. Oncol. 17(7), 2105-2116 (1999).

19 Ma X, Yan W, Zheng H et al. Regulation of IL-10 and IL-12 production and function in macrophages and dendritic cells. F1000Res. 4, pii:F1000 Faculty Rev-1465 (2015) (Epub ahead of print).

20 Floros T, Tarhini AA. Anticancer cytokines: biology and clinical effects of interferon- $\alpha 2$, interleukin (IL)-2, IL-15, IL-21, and IL-12. Semin. Oncol. 42(4), 539-548 (2015).

21 Lasek W, Zagozdzon R, Jakobisiak M. Interleukin 12: still a promising candidate for tumor immunotherapy? Cancer Immunol. Immunother. CII 63(5), 419-435 (2014).

22 Prochazkova J, Pokorna K, Holan V. IL-12 inhibits the TGF- $\beta$-dependent T cell developmental programs and skews the TGF- $\beta$-induced differentiation into a Th1-like direction. Immunobiology 217(1), 74-82 (2012).

23 Billerbeck E, Labitt RN, Vega K et al. Insufficient interleukin-12 signalling favours differentiation of human CD4(+) and CD8(+) T cells into GATA-3(+) and GATA-3(+) T-bet(+) subsets in humanized mice. Immunology 143(2), 202-218 (2014).

24 Kerkar SP, Goldszmid RS, Muranski P et al. IL-12 triggers a programmatic change in dysfunctional myeloid-derived cells within mouse tumors. J. Clin. Invest. 121(12), 4746-4757 (2011).

25 Rossowska J, Pajtasz-Piasecka E, Anger N et al. Cyclophosphamide and IL-12-transduced DCs enhance the antitumor activity of tumor antigen-stimulated DCs and reduce Tregs and MDSCs number. J. Immunother. Hagerstown Md 1997 37(9), 427-439 (2014).

26 Atkins MB, Robertson MJ, Gordon M et al. Phase I evaluation of intravenous recombinant human interleukin 12 in patients with advanced malignancies. Clin. Cancer Res. 3(3), 409-417 (1997). 
27 Hurteau JA, Blessing JA, DeCesare SL, Creasman WT. Evaluation of recombinant human interleukin-12 in patients with recurrent or refractory ovarian cancer: a gynecologic oncology group study. Gynecol. Oncol. 82(1), 7-10 (2001).

28 Leonard JP, Sherman ML, Fisher GL et al. Effects of single-dose interleukin-12 exposure on interleukin-12-associated toxicity and interferon-gamma production. Blood 90(7), 2541-2548 (1997).

29 Del Vecchio M, Bajetta E, Canova S et al. Interleukin-12: biological properties and clinical application. Clin. Cancer Res. 13(16), 4677-4685 (2007).

30 Rook AH, Wood GS, Yoo EK et al. Interleukin-12 therapy of cutaneous T-cell lymphoma induces lesion regression and cytotoxic T-cell responses. Blood 94(3), 902-908 (1999).

31 Duvic M, Sherman ML, Wood GS et al. A Phase II open-label study of recombinant human interleukin-12 in patients with stage IA, IB, or IIA mycosis fungoides. J. Am. Acad. Dermatol. 55(5), 807-813 (2006).

32 Little RF, Pluda JM, Wyvill KM et al. Activity of subcutaneous interleukin-12 in AIDS-related Kaposi sarcoma. Blood 107(12), 4650-4657 (2006).

33 Younes A, Pro B, Robertson MJ et al. Phase II clinical trial of interleukin-12 in patients with relapsed and refractory non-Hodgkin's lymphoma and Hodgkin's disease. Clin. Cancer Res. 10(16), 5432-5438 (2004).

34 van Herpen CM, Looman M, Zonneveld $\mathrm{M}$ et al. Intratumoral administration of recombinant human interleukin 12 in head and neck squamous cell carcinoma patients elicits a T-helper 1 profile in the locoregional lymph nodes. Clin. Cancer Res. 10(8), 2626-2635 (2004).

35 Mahvi DM, Henry MB, Albertini MR et al. Intratumoral injection of IL-12 plasmid DNA-results of a Phase I/IB clinical trial. Cancer Gene Ther. 14(8), 717-723 (2007).

36 Radny P, Caroli UM, Bauer J et al. Phase II trial of intralesional therapy with interleukin-2 in soft-tissue melanoma metastases. $B r$. J. Cancer 89(9), 1620-1626 (2003).

37 Weide B, Derhovanessian E, Pflugfelder A et al. High response rate after intratumoral treatment with interleukin-2: results from a Phase II study in 51 patients with metastasized melanoma. Cancer 116(17), 4139-4146 (2010).

38 Neumann E, Schaefer-Ridder M, Wang Y, Hofschneider PH. Gene transfer into mouse lyoma cells by electroporation in high electric fields. EMBO J. 1(7), 841-845 (1982).

39 Mir LM, Gehl J, Sersa G. Standard operating procedures of the electrochemotherapy: instructions for the use of bleomycin or cisplatin administered either systemically or locally and electric pulses delivered by the Cliniporator by means of invasive or non-invasive electrodes. Eur. J. Cancer (Suppl. 4), 14-25 (2006).

40 Isaka Y, Imai E. Electroporation-mediated gene therapy. Expert Opin. Drug Deliv. 4(5), 561-571 (2007).

41 Lampreht U, Kamensek U, Stimac M et al. Gene electrotransfer of canine interleukin 12 into canine melanoma cell lines. J. Membr. Biol. 248(5), 909-917 (2015).

42 Sin J-I, Park J-B, Lee IH et al. Intratumoral electroporation of IL-12 cDNA eradicates established melanomas by Trp2(180-188)-specific $\mathrm{CD}^{+}$CTLs in a perforin/granzyme-mediated and IFN- $\gamma$-dependent manner: application of Trp2(180-188) peptides. Cancer Immunol. Immunother. CII 61(10), 1671-1682 (2012).

43 Radkevich-Brown O, Piechocki MP, Back JB, Weise AM, Pilon-Thomas S, Wei W-Z. Intratumoral DNA electroporation induces anti-tumor immunity and tumor regression. Cancer Immunol. Immunother. CII 59(3), 409-417 (2010).

44 Cemazar M, Ambrozic Avgustin J, Pavlin D et al. Efficacy and safety of electrochemotherapy combined with peritumoral IL-12 gene electrotransfer of canine mast cell tumours. Vet. Comp. Oncol. 15(2), 641-654 (2017).

45 Heller L, Merkler K, Westover J et al. Evaluation of toxicity following electrically mediated interleukin-12 gene delivery in a B16 mouse melanoma model. Clin. Cancer Res. 12(10), 3177-3183 (2006).

-• Assessed the toxicity and response of the TAVO-EP construct in animal models. The study also included sham injection controls.

46 Cutrera J, King G, Jones P et al. Safe and effective treatment of spontaneous neoplasms with interleukin 12 electro-chemo-gene therapy. J. Cell Mol. Med. 19(3), 664-675 (2015).

47 Lucas ML, Heller R. IL-12 gene therapy using an electrically mediated nonviral approach reduces metastatic growth of melanoma. DNA Cell Biol. 22(12), 755-763 (2003).

48 Li S, Zhang X, Xia X. Regression of tumor growth and induction of long-term antitumor memory by interleukin 12 electro-gene therapy. J. Natl Cancer Inst. 94(10), 762-768 (2002).

- Demonstrated immune memory respones in animals treated with plasmid IL-12 with electroporation supporting the hypothesis that this approach is mediated by in situ tumor immunization.

49 Pavlin D, Cemazar M, Kamensek U, Tozon N, Pogacnik A, Sersa G. Local and systemic antitumor effect of intratumoral and peritumoral IL-12 electrogene therapy on murine sarcoma. Cancer Biol. Ther. 8(22), 2114-2122 (2009).

50 Li S, Zhang L, Torrero M, Cannon M, Barret R. Administration route- and immune cell activation-dependent tumor eradication by IL-12 electrotransfer. Mol. Ther. J. Am. Soc. Gene Ther. 12(5), 942-949 (2005).

51 Jacobson JM, Zheng L, Wilson CC et al. The safety and immunogenicity of an interleukin-12-enhanced multiantigen DNA vaccine delivered by electroporation for the treatment of HIV-1 infection. J. Acquir. Immune Defic. Syndr. 71(2), 163-171 (2016). 
52 Mpendo J, Mutua G, Nyombayire J et al. A Phase I double blind, placebo-controlled, randomized study of the safety and immunogenicity of electroporated HIV DNA with or without interleukin 12 in prime-boost combinations with an Ad35 HIV vaccine in healthy HIV-seronegative African adults. PLoS ONE 10(8), e0134287 (2015).

53 Kalams SA, Parker SD, Elizaga M et al. Safety and comparative immunogenicity of an HIV-1 DNA vaccine in combination with plasmid interleukin 12 and impact of intramuscular electroporation for delivery. J. Infect. Dis. 208(5), 818-829 (2013).

54 Meeting Library. A clinical trial for the safety and immunogenicity of a DNA-based immunotherapy in men with biochemically (PSA) relapsed prostate cancer. http://meetinglibrary.asco.org/record/145240/abstract

55 Meeting Library. Study of hTERT and IL-12 DNA immunotherapy using electroporation in patients with solid tumors after definitive surgery and adjuvant therapy. http://meetinglibrary.asco.org/record/115140/abstract

56 Algazi A, Tsai KK, Takamura KT et al. Abstract CT134: intratumoral electroporation of plasmid IL-12 can prime response to anti-PD1/PD-L1 blockade in patients with Stage III/IV-M1a melanoma. Cancer Res. 76(14 Suppl.), CT134 (2016).

57 Daud A, Algazi AP, Ashworth MT et al. Systemic antitumor effect and clinical response in a Phase II trial of intratumoral electroporation of plasmid interleukin-12 in patients with advanced melanoma. J. Clin. Oncol. 32(5s Suppl.), Abstract 9025 (2014).

-• Phase II study confirming that plasmid IL-12 with electroporation induces objective responses in patients including abscopal regressions of untreated lesions.

58 Daud AI, DeConti RC, Andrews $S$ et al. Phase I trial of interleukin-12 plasmid electroporation in patients with metastatic melanoma. J. Clin. Oncol. 26(36), 5896-5903 (2008).

-• First-in-human Phase I trial of tavo electroporation in patients with metastatic melanoma with accessible cutaneous disease that was surgically unresectable.

59 Tugues S, Burkhard SH, Ohs I et al. New insights into IL-12-mediated tumor suppression. Cell Death Differ. 22(2), 237-246 (2015).

60 Rakhmilevich AL, Timmins JG, Janssen K, Pohlmann EL, Sheehy MJ, Yang NS. Gene gun-mediated IL-12 gene therapy induces antitumor effects in the absence of toxicity: a direct comparison with systemic IL-12 protein therapy. J. Immunother. Hagerstown Md 1997. 22(2), 135-144 (1999).

61 Lohr F, Lo DY, Zaharoff DA et al. Effective tumor therapy with plasmid-encoded cytokines combined with in vivo electroporation. Cancer Res. 61(8), 3281-3284 (2001).

62 Lucas ML, Heller L, Coppola D, Heller R. IL-12 plasmid delivery by in vivo electroporation for the successful treatment of established subcutaneous B16.F10 melanoma. Mol. Ther. J. Am. Soc. Gene Ther. 5(6), 668-675 (2002).

63 Garcia-Diaz A, Shin DS, Moreno BH et al. Interferon receptor signaling pathways regulating PD-L1 and PD-L2 expression. Cell Rep. 19(6), 1189-1201 (2017).

64 Daud AI, Wolchok JD, Robert C, Hwu W-J, Weber JS, Ribas A et al. Programmed death-ligand 1 expression and response to the anti-programmed death 1 antibody pembrolizumab in melanoma. J. Clin. Oncol. 34(34), 4102-4109 (2016).

- This paper demonstrated that the inflammatory marker PD-L1 immunohistochemistry could be quantified to predict antitumor immune response to PD-1 antibodies in melanoma underscoring the importance of intratumoral inflammation in antitumor immune responses.

Clinical trials database: NCT01502293. www.clinicaltrials.gov/ct2/show/NCT01502293?term=electroporation $+i l-12 \&$ draw $=1 \& \mathrm{rank}=3$

Clinical trials database: NCT03132675. www.clinicaltrials.gov/ct2/show/NCT03132675?term=pIL12-EP+pembrolizumab\&rank=1 
